\title{
NONISOMORPHIC APPROXIMATELY FINITE FACTORS
}

\section{HAROLD WIDOM}

In [2] two notions of approximate finiteness were introduced for finite factors on (possibly inseparable) Hilbert space. The question was left open whether these two notions, approximate finiteness (A) and (B), are distinct. The purpose of this paper is to show that they are. Recall that a factor $\boldsymbol{M}$ of type $\mathrm{II}_{1}$ is approximately finite (A) if given $A_{1}, \cdots, A_{n} \in M$ and $\epsilon>0$, there exists a subfactor $N$ of $\boldsymbol{M}$ which is of type $I$ and contains elements $B_{1}, \cdots, B_{n}$ satisfying $\left[\left[B_{i}-A_{i}\right]\right]<\epsilon(i=1, \cdots, n) ; M$ is approximately finite (B) if it contains mutually commuting subfactors $N_{\alpha}$ of type I such that $\mathrm{V} N_{\alpha}=M$ (where " $\mathrm{V}$ " means "ring generated by").

Before beginning the work necessary in proving the existence of factors approximately finite (A) but not (B), we should like to outline the construction of factors of type $\mathrm{II}_{1}$ from groups. Our notation will be different from that of $[1$, Chapter 5$]$, where this construction was introduced.

Let $G$ be a (discrete) group, $H$ the Hilbert space of all complex valued functions $g \rightarrow x(g)$ such that $\sum_{o \in G}|x(g)|^{2}<\infty$. For $g \in G$ let $U_{0}$ be the unitary operator on $H$ defined by $U_{0} x\left(g^{\prime}\right)=x\left(g g^{\prime}\right)$, and define $\mathscr{L}(G)$ to be the ring of operators generated by the various $U_{0}$. If $G$ has the property that all its nontrivial conjugate classes are infinite, then $\mathscr{L}(G)$ is a factor of type $\mathrm{II}_{1}$.

For any $x, y \in H$ define $x * y$ by $x * y(g)=\sum_{h \in G} x(h) y\left(h^{-1} g\right)$, and let $H^{\prime}$ be the set of all $x \in H$ such that the map $y \rightarrow x * y$ is a bounded operator on $H$. Then we have $\mathfrak{L}(G)=\left\{U_{x}: x \in H^{\prime}\right\}$, where $U_{x}$ is the operator $y \rightarrow x * y$. Moreover we have $U_{x} U_{y}=U_{x^{*} y}$, and the trace on $\mathfrak{L}(G)$ is given by $T\left(U_{x}\right)=x(e), e$ being the identity element of $G$.

Finally let $G_{0}$ be a subgroup of $G$. Then $\mathscr{L}\left(G, G_{0}\right)=\mathrm{V}\left\{U_{0}: g \in G_{0}\right\}$ is a subring of $\mathscr{L}(G)$ which is naturally isomorphic with $\mathscr{L}\left(G_{0}\right)$.

Call $G$ locally finite if any finite subset of $G$ generates a finite subgroup. Then by the method of $\$ 4.6$ and $\$ 5.6$ of [1] one can easily prove the following.

LEMмA 1. If $G$ is locally finite and has infinite nontrivial conjugate classes, then $\mathfrak{L}(G)$ is approximately finite $(A)$.

In the next lemma we use the notation $\chi(\boldsymbol{M})$, for a finite factor $\boldsymbol{M}$, to denote the density character of $M$ relative to the metric [[ ]].

Presented to the Society, October 27, 1956; received by the editors August 17, 1956. 
LEMMA 2. Let $G$ be a group with infinite nontrivial conjugate classes, $\aleph$ an infinite cardinal, and $\bar{G}$ the weak direct product of $\boldsymbol{\aleph}$ copies of $G$. Then if $\boldsymbol{M}=\mathfrak{L}(\bar{G})$ and $N$ is a subfactor of $\boldsymbol{M}$ with $\chi(N)<\boldsymbol{N}$, the ring $N^{\prime} \cap M$ (the commutor of $N$ in $M$ ) is larger than the complex numbers.

Proof. The general element of $\bar{G}$ will be denoted by $\left\{g_{i}\right\}$, where $i$ runs through an index set $I$ of cardinality $\aleph$. Let $\left\{A_{\alpha}\right\}$ be a [[]]dense subset of $N$ with cardinality $\chi(N)$. Each $A_{\alpha}=U_{x_{\alpha}}$ for some $x_{\alpha} \in H^{\prime}\left(H^{\prime}\right.$ being obtained from $\left.\bar{G}\right)$. Since each $x_{\alpha}(\cdot)$ has countable support, the union $S$ of the supports of the $x_{\alpha}(\cdot)$ has cardinality $\boldsymbol{\aleph}_{0} \cdot \chi(N)=\chi(N)$. Moreover for each $\left\{g_{i}\right\} \in S, g_{i}=e$ (the identity of $G)$ for all but finitely many $i \in I$. Thus the set

$$
J=\bigcup_{\{0 i\} \in S}\left\{i \in I: g_{i} \neq e\right\}
$$

has cardinality $\chi(\boldsymbol{N})$, which is less than the cardinality of $I$. Therefore we can find an $i_{0} \in I-J$. Let $\bar{g}$ be an element of $\bar{G}$ whose $i$ th component is $e$ for $i \neq i_{0}$, and arbitrary but $\neq=e$ for $i=i_{0}$. Then $\bar{g}$ commutes with every element of $S$, so $A=U_{g}$ commutes with each $A_{\alpha}$ and so with $N$.

The following result gives a distinguishing characteristic of factors approximately finite (B).

THEOREM 1. Let $M$ be approximately finite (B) and $N$ a subfactor of $\boldsymbol{M}$ satisfying $\chi(\boldsymbol{N})<\chi(\boldsymbol{M})$. Then $\boldsymbol{N}^{\prime} \cap \boldsymbol{M}$ is larger than the complex numbers.

Proof. Let $G_{0}$ be the group of those permutations of a denumerable set which move only finitely many elements, and $G$ the weak direct product of $\chi(M)$ copies of $G_{0}$. As was shown in $\S 5$ of $[2], \mathcal{L}(G)$ is approximately finite (B) with density character $\chi(\boldsymbol{M})$. But then by Theorem 2 of [2], $M$ and $\mathcal{L}(G)$ are isomorphic. The result is now an obvious consequence of Lemma 2.

We now go in the reverse direction, producing subfactors with only trivial commutors.

Lemma 3. Let $G$ be a group possessing a subgroup $G_{0}$ such that for every element $g \in G$ other than the identity, the set $\left\{g_{0} g g_{0}^{-1}: g_{0} \in G_{0}\right\}$ is infinite. Then $\mathscr{L}\left(G, G_{0}\right)^{\prime} \cap \mathcal{L}(G)$ is exactly the complex numbers.

Proof. Let $A=U_{x} \in \mathfrak{L}\left(G, G_{0}\right)^{\prime} \cap \mathfrak{L}\left(G_{0}\right)$. We must show that $x$ vanishes everywhere except possibly at $e$. Let $g_{0} \in G_{0}$ be arbitrary and define $y \in H^{\prime}$ by $y(g)=0$ for $g \neq g_{0}^{-1}$ and $y\left(g_{0}^{-1}\right)=1$. Then $U_{\nu}=U_{g_{0}}$ $\in \mathcal{L}\left(G, G_{0}\right)$ so we have $x * y=y * x$, i.e. $x\left(h g_{0}\right)=x\left(g_{0} h\right)$ for all $h \in G$. Letting $h=g g_{0}^{-1}$, we have $x(g)=x\left(g_{0} g g_{0}^{-1}\right)$ for all $g \in G$. But if $g \neq e$ the 
set $\left\{g_{0} g g_{0}^{-1}: g_{0} \in G_{0}\right\}$ is infinite, and yet $\sum_{o \in G}|x(g)|^{2}<\infty$. Therefore we must have $x(g)=0$ for $g \neq e$.

We proceed to construct groups to which Lemma 3 can be applied. Let $\boldsymbol{\aleph}$ be an infinite cardinal and $G_{0}$ the group of all permutations of a denumerable set which move only finitely many elements. Form the full direct product of $\aleph$ copies of $G_{0}$, the general element of the direct product being denoted by $\left\{g_{i}\right\}$. Now let $\bar{G}_{1}$ be the subgroup of this direct product consisting of all $\left\{g_{i}\right\}$ such that there are only finitely many distinct $g_{i}$ 's. Any finite subset of $\bar{G}_{1}$ can be embedded in a subgroup isomorphic to a finite direct product of copies of $G_{0}$; since $G_{0}$ is locally finite this shows that $\bar{G}_{1}$ is also locally finite. The group $G_{0}$ is naturally isomorphic to the diagonal $\bar{G}_{0}$ of $\bar{G}_{1}$. Since $\bar{G}_{1}$ is locally finite and has cardinality $2^{\text {N }}$ we can find a subgroup $\bar{G}$ of $\bar{G}_{1}$ which contains $\bar{G}_{0}$ and has cardinality $\boldsymbol{\aleph}$. Let $\bar{g}=\left\{g_{i}\right\}$ be an element of $\bar{G}$ other than the identity, say $g_{j} \neq e$. For any $g_{0} \in G_{0}$ let $\bar{g}_{0}$ be the element of $\bar{G}_{0}$ all of whose components are $g_{0}$. Then $\bar{g}_{0} \bar{g} \bar{g}_{0}^{-1}=\left\{g_{0} g_{i} g_{0}^{-1}\right\}$. But $\left\{g_{0} g_{j} g_{0}^{-1}: g_{0} \in G_{0}\right\}$ is infinite since $g_{j} \neq e$. Thus the set of $j$ th components of $\left\{\bar{g}_{0} \bar{g} \bar{g}_{0}^{-1}: \bar{g} \in \bar{G}_{0}\right\}$ is infinite, so the set itself certainly is. Now removing the bars from the groups $\bar{G}$ and $\bar{G}_{0}$ we may sum up the above discussion in

Lemma 4. Given an infinite cardinal $\aleph$ we can find a locally finite group $G$ having that cardinality and possessing a denumerable subgroup $G_{0}$ such that $e \neq g \in G$ implies that the set $\left\{g_{0} g g_{0}^{-1}: g_{0} \in G_{0}\right\}$ is infinite.

We can now prove the main result of the paper.

Theorem 2. Let $\boldsymbol{\aleph}$ and $\boldsymbol{\aleph}^{\prime}$ be infinite cardinals with $\boldsymbol{\aleph}^{\prime} \leqq \boldsymbol{N}$. Then we can find a factor $\boldsymbol{M}$ which is approximately finite (A) and such that moreover

(a) $\chi(M)=\mathbf{N}$,

(b) if $\boldsymbol{N}$ is a subfactor of $M$ with $\chi(N)<\boldsymbol{N}^{\prime}$ then $\boldsymbol{N}^{\prime} \cap M$ is larger than the complex numbers,

(c) there exists a subfactor $\boldsymbol{N}$ of $\boldsymbol{M}$ with $\chi(\boldsymbol{N})=\boldsymbol{N}^{\prime}$ such that $\boldsymbol{N}^{\prime} \cap M$ is exactly the complex numbers.

Proof. Starting with the groups $G$ and $G_{0}$ of Lemma 4 , let $\bar{G}$ be the weak direct product of $\boldsymbol{\aleph}^{\prime}$ copies of $G$ and $\bar{G}_{0}$ the subgroup of $\bar{G}$ which is the weak direct product of $\boldsymbol{\aleph}^{\prime}$ copies of $G_{0}$. We see immediately that $\bar{G}$ is locally finite and that for any element $\bar{g}$ of $\bar{G}$ other than the identity the set $\left\{\bar{g}_{0} \bar{g} \bar{g}_{0}^{-1}: \bar{g}_{0} \in \bar{G}_{0}\right\}$ is infinite. In particular $\bar{G}$ has infinite nontrivial conjugate classes. Thus by Lemma $1, M=\mathscr{L}(\bar{G})$ is an approximately finite (A) factor of type $\mathrm{II}_{1}$. Moreover since $\bar{G}$ has cardinality $\boldsymbol{N}$, (a) is immediate. Again, if $N=\mathfrak{L}\left(\bar{G}, \bar{G}_{0}\right)$ then 
$\chi(\boldsymbol{N})=\boldsymbol{\aleph}^{\prime}$ since $\bar{G}_{0}$ has cardinality $\boldsymbol{\aleph}^{\prime}$, and (c) follows from Lemma 3 . (Note that since $\bar{G}_{0}$ has infinite nontrivial conjugate classes, $N$ is a subfactor of type $\mathrm{II}_{1}$.) Finally (b) follows from Lemma 2.

Corollary 1. Given any $\boldsymbol{\aleph}>\boldsymbol{\aleph}_{0}$ we can find a type $\mathrm{II}_{1}$ factor $\boldsymbol{M}$ with $\chi(\boldsymbol{M})=\boldsymbol{N}$ such that $\boldsymbol{M}$ is approximately finite (A) but not (B).

Proof. Let $\boldsymbol{M}$ be the factor of Theorem 2 corresponding to any infinite $\boldsymbol{N}^{\prime}<\boldsymbol{N}$. Then Theorems 1 and 2 show $\boldsymbol{M}$ is approximately finite (A) but not (B).

Corollary 2. Given a cardinal $\boldsymbol{N}_{\omega} \geqq \boldsymbol{N}_{0}$ there exist at least $|\omega|+1$ nonisomorphic factors which are approximately finite (A) and have density character $\boldsymbol{\aleph}_{\omega}$.

Proof. The factors of Theorem 2 corresponding to different $\boldsymbol{\aleph}^{\prime}$ are nonisomorphic, and there are $|\omega|+1$ possibilities for $\boldsymbol{\aleph}^{\prime}$.

\section{REFERENCES}

1. F. J. Murray and J. von Neumann, On rings of operators IV, Ann. of Math. vol. 44 (1943) pp. 716-808.

2. H. Widom, Approximately finite algebras, Trans. Amer. Math. Soc. vol. 83 (1956) pp. 170-178.

Cornell University 